\title{
Physical sciences dominate Japan's special grant awards
}

Tokyo. The physical sciences and technology have moved ahead of biology on this year's list of grants to individual scientists announced last week by the Japanese Ministry of Education, Science and Culture. Researchers in physics, chemistry and astronomy received seven of the 11 awards in the ministry's 'special distinguished' grant category, a competition that has been dominated by the life sciences during most of the past decade.

The grants, worth about $¥ 100$ - $¥ 300$ million (US\$750,000-\$2.2 million) over three to five years, are meant to support "internationally renowned research that is likely to give outstanding results". They provide an indication of where some of Japan's best basic research is being done.

One of the largest grants — $¥ 283$ million for five years - goes to Toshimitsu Yamazaki of the Institute for Nuclear Study of Tokyo University. Yamazaki will use the low-energy antiproton beam at the European high-energy physics laboratory (CERN) in Geneva to study anomalously long-lived hadronic atoms, such as antiprotons embedded in helium. The grant will further strengthen the collaboration between CERN and Japan's leading national university.

Daiichiro Sugimoto of Tokyo University's department of Earth science and astronomy, who has developed a high-speed computer to model evolution of star clusters and galaxies (Nature 345, 33; 1990), gets $¥ 189$ million for five years to upgrade his computer to carry out teraflop calculations of such many-body problems. Sugimoto intends to model not only the behaviour of stars and galaxies but also the molecular dynamics of, for example, proteins.

Another user of super-high-speed technology is Akira Hasegawa of Osaka University's department of communications engineering. He receives $¥ 185$ million over five years to test an optical fibre transmission system based on the idea that solitons can transmit vast amounts of multichannel data (40 gigabits) over tens of thousands of kilometres.

In contrast with previous years, only four awards went to those in the biological sciences. But two of them are among the largest. Tasuku Honjo of Kyoto University receives $¥ 184$ million over three years to elucidate the molecular and regulatory mechanism of programmed cell death, an important element in understanding embryogenesis and autoimmune diseases. And Nobutaka Hirokawa of Tokyo University faculty of medicine receives the largest grant — $¥ 290$ million for four years - to study the molecular architecture, dynamics and function of the neuronal cytoskeleton.

David Swinbanks
NEWS IN BRIEF

Washington. The European space probe Giotto has been put to electronic sleep and is heading back to Earth after its successful encounter last month with comet Grigg-Skjellerup. But the seven-yearold-spacecraft, which flew by comet Halley in 1986, has probably carried out the last of its scientific duties.

Although Giotto is projected to come within $220,000 \mathrm{~km}$ of Earth in 1999, it is unlikely to pass near another comet. Even if it did, it probably lacks the fuel to manoeuvre into position to make observations. Finally, European Space Agency officials are not sure that they can afford to maintain for the rest of the decade the groundbased hardware used to communicate with Giotto.

J.M.

New Delhi. The Indian government says that the Russian space agency has promised to honour a contract to purchase cryogenic rocket engines that has triggered a boycott by the United States.

"The contract between ISRO [Indian Space Research Organization] and Glavkosmos remains in force and is being implemented," India's science minister, P.R. Kumaramangalam, told parliament last week. He said that Russian officials have "reassured" India that they will carry out the contract. India has already paid Russia more than a third of the $\$ 80$ million it will cost to acquire two engines and the accompanying technology.

The United States believes that the contract violates an international agreement to prevent the spread of nuclear weapons technology, but India and Russia insist that the technology will be used only for civilian purposes. The rockets will be part of a geostationary launch vehicle to be tested in 1995.K.S.J.

Washington. Denmark's Medical Research Council has released the country's first plan for dealing with allegations of scientific misconduct and ethical violation in research. Detailing eight well-known cases of misconduct in the United States and other countries (but none in Denmark), the council said that a contributing factor was the lack of established procedures for dealing with such abuses in those countries. It recommended new guidelines on clinical research ethics and conflict of interest, and the creation of a national committee to review charges of misconduct.

The committee, which will be functioning by October, will have a high court judge as chairman and six health science researchers as members. When appropriate, the committee is expected to set up special ad hoc committees to investigate specific cases. The report also recommends that scientists under investigation be given the right to representation by an attorney, appeal. cross-examination and examination of the data. tists working out of either the White House or the US National Institutes of Health to set fornia, Berkeley, who believes that Clinton win the election. "They've let it drop, and now it's gone."
Traci Watson 